\title{
IMPLEMENTATION OF AUTOMATED MOTOR STARTER UNIT FOR SMART FARMING IN INDIA
}

\author{
A PREPRINT \\ Karthik Muthineni ${ }^{*}$ \\ Department of Electronics and Communication Engineering \\ Jawaharlal Nehru Technological University \\ Hyderabad, India 500085 \\ karthikm618@gmail.com \\ Akhil Yalagonda \\ Department of Electronics and Communication Engineering \\ Jawaharlal Nehru Technological University \\ Hyderabad, India \\ Praveen Gorla \\ Department of Electronics and Communication Engineering \\ Jawaharlal Nehru Technological University \\ Hyderabad, India \\ Tarun Pulluri \\ Department of Electronics and Communication Engineering \\ Jawaharlal Nehru Technological University \\ Hyderabad, India
}

June 29,2020

\begin{abstract}
Agriculture is one of the key enabler for the economic development of India that accounts for one third of nation's income. Due to the problems that agriculture industry is facing, there is a need to introduce automation in agriculture that improves the efficiency. This paper presents the implementation of Automated Motor Starter Unit (AMSU) for smart farming applicable to indian scenario. In addition, current technologies and attempts in smart farming are reviewed and discussed. More than $90 \%$ of indian farmers have been using motors for their farm fields which are controlled by single phase or three phase power supplies. The AMSU has been designed to turn on/off motor in the farm field using mobile phone having cellular network from any place. The AMSU is selected to increase the operation efficiency by minimizing the manual operation of motors for which farmer need to go to the farm field.
\end{abstract}

Keywords Motor Starter Unit · Single Phase · Three Phase

\footnotetext{
${ }^{*}$ Currently holds Researcher position in the Cyber Physical Systems Research Unit, National Electronics and Computer Technology Center, Thailand. Between February 2019 and March 2020, he has served as lead developer for the AIT OpenCellular project, sponsored by Facebook. His research interests include Wireless Sensor Networks, Indoor Localization, and Cyber Physical Systems.
} 


\section{Introduction}

Agriculture is the main source of food grains and plays an important role in the economic development of agricultural countries. India is a agriculture country with $70 \%$ of population depending on agriculture or related fields. Income generated from agriculture accounts for the one third of nation's capital [1, 2]. However, this is one side of the coin, the other side contain several problems that decreases the production yield due to inadequate rainfalls, uncertainty in power supply, and traditional methods followed by farmers. The primary requirement for any crop growth is the sufficient and uninterrupted water supply. In many parts of the country, due to inadequate rainfalls the irrigation systems were developed that stores the water during heavy rainfall and releases to the farm field for the crop growth whenever required using single phase or three phase motors that need to be operated manually by farmer. Usually the distance from farm field to water source ranges in few kilometers and farmer need to walk all the way in order to operate the motor. In some cases, due to insufficient water present in well, motor runs in dry state and leads to complete failure. And, fluctuations in electrical supply resulting in voltage variation of higher than $\pm 10 \%$ of prescribed nameplate rating also leads to motor failure [3, 4]. All the above stated reasons will significantly decrease the efficiency of agriculture production.

Integration of modern technologies with agricultural methods will reduce the burden on farmer and improves overall efficiency. Wireless Sensor Network (WSN) consist of sensors and actuators to monitor different parameters and act accordingly. One of the best application of WSN is in agriculture domain where different parameters are monitored and communicated wirelessly to take appropriate actions. In general WSN applications in agriculture domain consist of sensor nodes, gateway that collects the data and sends to cloud for processing. Such applications are in demand and impose challenges in real time implementation. Some of such challenges are low cost, power requirement, and ease of use for farmers. Terrestrial Wireless Sensor Network (TWSN) and Wireless Underground Sensor Network (WUSN) are the two most commonly used networks in agriculture domains [5]. In TWSN, sensor nodes are placed above the surface of ground and will communicate with gateway using Industrial, Scientific, Medical (ISM) bands of 902-928 MHz and $2.4 \mathrm{GHz}$. On other hand, WUSN uses sensors that are deployed under the surface of ground. Soil moisture sensor used to measure soil wetness is one of the example for WUSN. Short range wireless communication is used between sensor nodes and gateway. IEEE 802.15.4 Zigbee, IEEE 802.15.1 Bluetooth, and IEEE 802.15.6 Ultra Wide Band (UWB) are few examples [6, 7, 8]. The communication between gateway and remote desktop supports long range for which IEEE $802.11 \mathrm{~b} / \mathrm{g}$ Wireless Local Area Network (WLAN) are used.

In this paper, we present an low cost TWSN implementation of Automated Motor Starter Unit (AMSU) that monitors the water level in well and sends SMS to farmer's mobile using Global System for Mobile Communication (GSM), depending on the status of the water level farmer can turn on/off motor using his mobile by sending SMS or giving a missed call to the number in the AMSU. In past few years, advances in agriculture has gained interest among several researchers who came up with different methods, that are presented in Table 1.

This paper is organized as follows. Section 2 presents different wireless communication technologies available with their features. Section 3 presents the complete implementation of AMSU for real time implementation in agriculture environment. This section also presents the hardware details for the prototype. Section 4 concludes the paper. 
Table 1: Summary of wireless applications in agriculture.

\begin{tabular}{lll}
\hline Category & Description & Reference \\
\hline GSM & $\begin{array}{l}\text { Wireless communication of temperature, humidity, } \\
\text { and wind speed using GSM-SMS }\end{array}$ \\
Automated water irrigation system for crop growth & using GSM \\
Implementation of green house environment \\
monitoring system with zigbee using CC2530 chip \\
Zigbee
\end{tabular}

\section{Wireless Communication Protocols}

Till now there is no such protocol dedicated to the agriculture applications. But over past few years, several short range and long range communication protocols were implemented by agriculture applications by researchers which shown promising results. Summary of such such protocols are presented in Table 2

\subsection{Cellular}

Cellular communication supports high data rate by utilizing GSM, 3G, and 4G capabilities. GSM is the most commonly used cellular communication in agriculture applications due to its Short Message Service (SMS) feature. It has uplink frequency of 890-915 MHz and downlink frequency of 935-960 MHz. It has bandwidth of $25 \mathrm{MHz}$ and uses Gaussian Minimum Shift Keying (GMSK) modulation [18].

\subsection{Zigbee}

Zigbee is used for the applications that need short range communication of about $10 \mathrm{~m}$. In agriculture, zigbee is used as an transreceiver to transmit the sensor data to the nearby gateway node. It uses IEEE 802.15.4 standard, basically developed for low power consumption and low data rate applications. It uses $5 \mathrm{MHz}$ bandwidth and Carrier Sense Multiple Access (CSMA) that avoids multiple data transmission at a time to the receiver [19]. Each transmitter in the network checks for the carrier signal from another transmitter, if it has found one then it waits till the other transmitter finishes its transmission. This mechanism avoids the collision between two signals.

\subsection{Bluetooth}

Bluetooth is a IEEE 802.15.1 standard used for most of the Internet of Things (IoT) applications. It is an terrestrial short range communication technology that operates using $2.4 \mathrm{GHz}$ frequency band. It consist of three classes namely Class 1, Class 2, and Class 3 with 100m, 10m, and 1m ranges. It uses Differential Phase Shift Keying (DPSK) and GFSK modulation techniques [20]. 
Table 2: Summary of Wireless Protocols.

\begin{tabular}{lllll}
\hline Protocol & Frequency Band & Data Rate & Power & Range \\
\hline Cellular & $\begin{array}{l}890-915 \mathrm{MHz}, \\
935-960 \mathrm{MHz}\end{array}$ & High & High & $35 \mathrm{kM}$ \\
$\begin{array}{l}\text { Zigbee } \\
\text { (IEEE 802.15.4) }\end{array}$ & $2.4 \mathrm{Ghz}$ & $250 \mathrm{Kbps}$ & Low & $10 \mathrm{~m}$ \\
$\begin{array}{l}\text { Bluetooth } \\
\text { (IEEE 802.15.1) }\end{array}$ & $2.4 \mathrm{GHz}$ & $2 \mathrm{Mbps}$ & Low & $10-100 \mathrm{~m}$ \\
$\begin{array}{l}\text { Wi-Fi } \\
(\text { IEEE 802.11b/g) }\end{array}$ & $2.4 \mathrm{GHz}, 5 \mathrm{GHz}$ & $11 \mathrm{Mbps}$ & High & $100 \mathrm{~m}$ \\
$\begin{array}{l}\text { LoRaWAN } \\
\text { (IEEE 802.11ah) }\end{array}$ & $923 \mathrm{MHz}$ & $37.5 \mathrm{Kbps}$ & Very Low & $5 \mathrm{Km}$ \\
\hline
\end{tabular}

\subsection{Wi-Fi}

$\mathrm{Wi}-\mathrm{Fi}$ is the most commonly used terrestrial communication protocol in applications that require long range. It uses both $2.4 \mathrm{GHz}$ and $5 \mathrm{GHz}$ and comes with different variations like IEEE $802.11 \mathrm{a} / \mathrm{b} / \mathrm{g} / \mathrm{n}$. Using Wi-Fi, signal can be transmitted to a distance of greater than $120 \mathrm{~m}$ with a data rate of greater than $11 \mathrm{Mbps}$. It uses Orthogonal Frequency Division Multiplexing (OFDM), Quadrature Amplitude Modulation (QAM), and Phase Shift Keying (PSK) modulation techniques [21].

\subsection{LoRaWAN}

LoRaWAN is a IEEE802.11ah standard used in applications that require large area coverage with low power consumption. It operates on LoRa network and has channel bandwidth of $125 \mathrm{KHz}$ to $500 \mathrm{KHz}$. It supports data rate of of $37.5 \mathrm{Kbps}$. In LoRaWAN all the transmitters can transmit signals simultaneously, there is no collision avoidance mechanism [22].

\section{AMSU Implementation for Agriculture}

In this section, we describe the system model for the AMSU prototype. The goal of the AMSU is to overcome the manual operation of motor in the farm field by automating the process and alert the farmer regarding water status in the well. The AMSU prototyping system is presented in Figure 3 To determine the water level in the well float switch is used. A float switch consist of internal float that moves up and down due to liquid flow in which it is placed. The internal float consist of reed switch and magnet that comes two positions namely Normally Closed (NC), Normally Opened (NO). Due to liquid flow, the reed switch operates either in "NC" or "NO" position thus closing or opening an electric circuit which thereby sends an output signal. Float switches comes in two types, horizontal or vertical. In this prototype, we have used the vertical float switch that is covered with steel net to prevent from dust as shown in Figure 1 . The other end of the float switch is connected to the AMSU. The AMSU unit consist of microcontroller, transreceiver, and relay circuit. The relay circuit is connected with the Control Unit (CU). The CU is an three phase motor switch panel with START/STOP buttons used to manually control the motor in the farm field. Along with START/STOP buttons, the CU consist of Fuses, Contactor, and Relays. In general, the three phase induction motor draws high current that can damage the motor windings. To protect the motor from over current fuses are used. Whenever there is a high current flow than the prescribed rating, the fuses will open the terminals. A Contactor consist of an coil that energizes when current flows through it, thus creating the magnetic field, which in turn causes the START/STOP buttons to close or open the electric circuit. There are cases in which the load connected to the motor draws high current, causing overload problem. To protect the motor from overload failure, relays are used that will cut the current flow during overload. The wiring of three phase motor with START/STOP buttons is shown in Figure 2 To remotely the control the AMSU through mobile, the GSM link is used. Farmer can turn motor on/off buy giving an missed call or by sending a SMS to the number installed in the AMSU. Only farmer and authorized users by farmer can control the AMSU, thus providing security. 


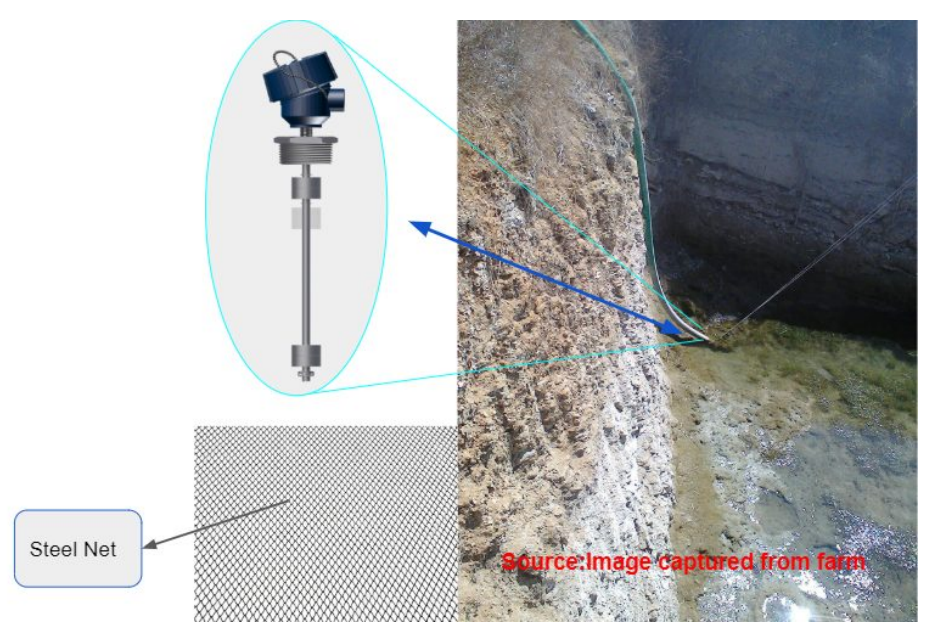

Figure 1: Float sensor installation.

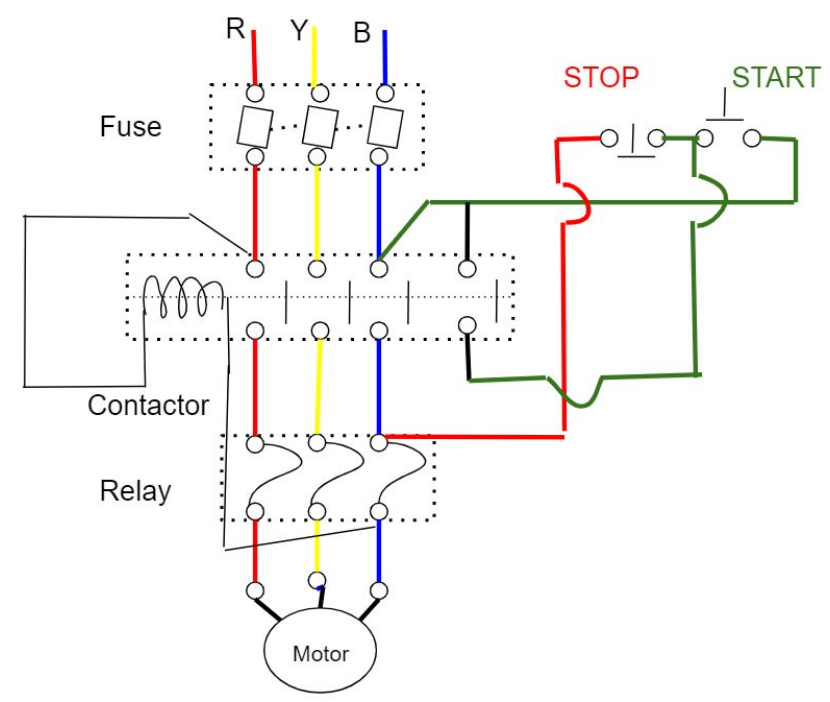

Figure 2: Wiring of three phase motor. 


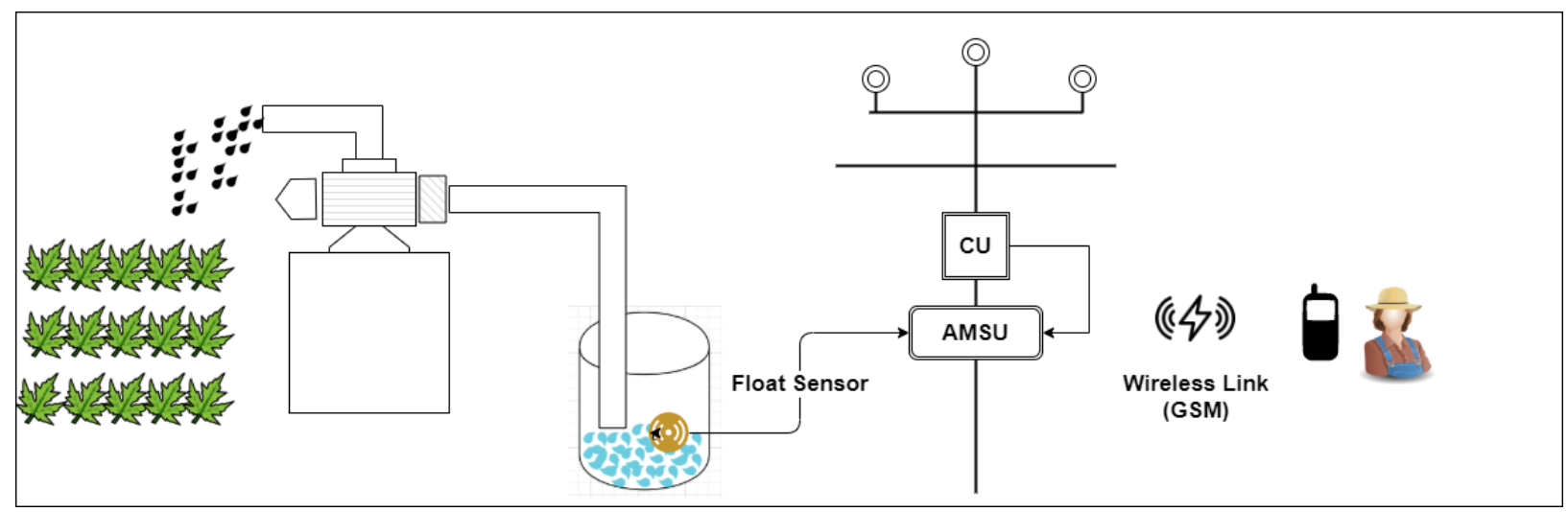

Figure 3: System Model.

\subsection{Hardware design}

In our AMSU prototype system we used microcontroller, GSM shield, relay circuit, and float switch. We have used Arduino Pro mini as microcontroller which is based on ATmega328. It operates on $3.3 \mathrm{~V}$ and consist of fourteen digital input/output pins, six analog input pins. SIM900A is used as GSM shield that operates in dual band 900/1800 MHz. It is used in voice, SMS, data, and low power consumption applications. It consist of sim holder for placing sim card, antenna, General Purpose Input Output (GPIO) pins, serial interface, $12 \mathrm{~V}$ battery slot, and Analog to Digital Converter (ADC). The relay circuit consist of 250 VAC 10 A ice cube relays, LM7805 voltage regulator, $1000 \mu \mathrm{F} 25 \mathrm{~V}$ Aluminum Electrolytic Capacitor, $470 \mu \mathrm{F} 25 \mathrm{~V}$ Aluminum Electrolytic Capacitor, IN4007 diodes, and prototype Printed Circuit Board (PCB) 4" x 2". A vertical float switch is used to detect the water level. The prototype model is shown in Figure 4

\subsection{Working Model}

- AMSU is installed at the field level by connecting it to the CU which is already connected to the three phase or single phase motor in the field.

- Four wires from AMSU are connected to existing CU (by opening the case cover ) to automate its performance.

- The present module works with line 2 (L2) supply and ground, which provides 230 VAC which is stepped down to 12 V 3 A supply which is fed to the Chipset.

- Power to the AMSU module is supplied by connecting it to the line (L2) of three phase supply which is already given to the existing CU.
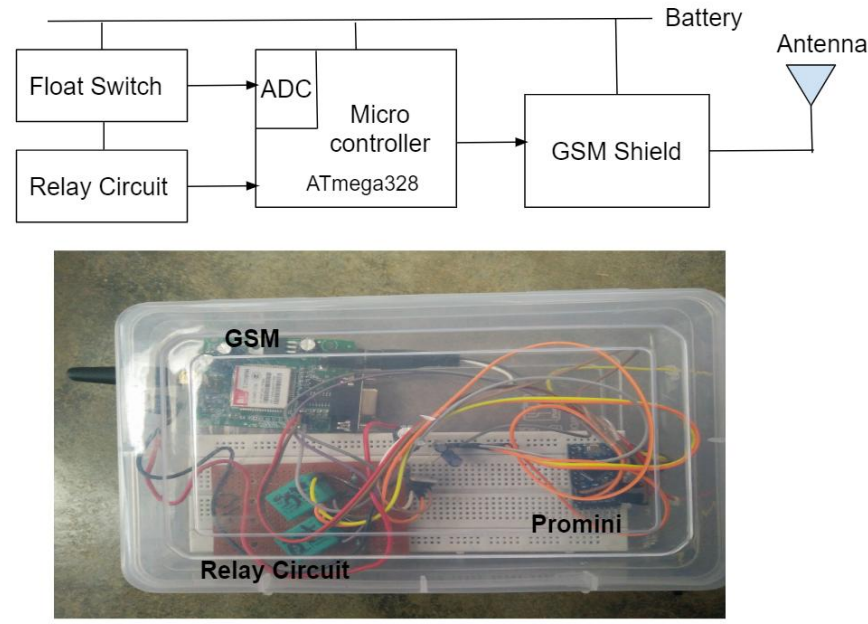

Figure 4: Sensor node hardware design. 
- There is an optional availability of two wires from AMSU to connect the float switch to find the availability of water level in the well.

- Only registered contact numbers can get access to the AMSU.

- A registered contact can add any number of contacts to operate it. (Limited to SIM memory)

- Once a number is registered and the power in the farm field is available, then AMSU will automatically send a message about the POWER and WATER STATUS to the registered mobile numbers.

- The AMSU can be operated by an SMS or MISSED CALL to the Number that is placed in AMSU.

- If water is not available in the well then the AMSU will detect it through the float switch and turns off the motor. A message is sent to the farmer mobile, stating that motor is off and water is not available (here AMSU will continuously read the status of the water level and keep itself updated). If farmer tries to switch on the motor without sufficient amount of water in the well i.e. about 5 inches, AMSU will not switch on the motor. Figure 5 shows the flowchart.

\section{Advantages}

- Save time by avoiding travelling to motor room every time to switch on and off motor.

- Save electricity by turning motor off while not needed.

- Save life by avoiding attack of wild animal while going to motor room in night time.

- Protect motor from dry run.

- Flexible wiring kit in modular way helps to make easy wiring and handling.

- Protect motor from phase imbalance and low voltage.

(a)

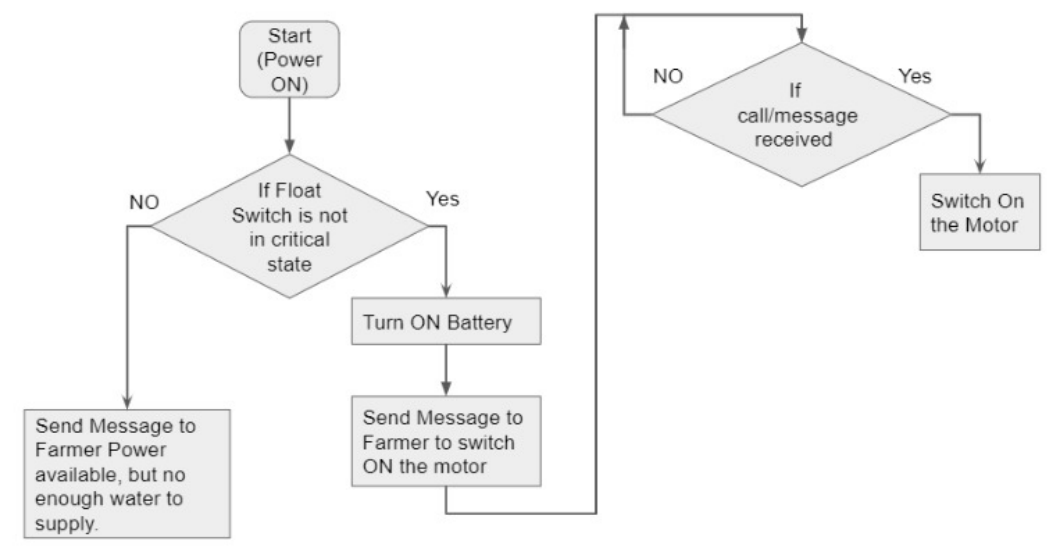

(b)

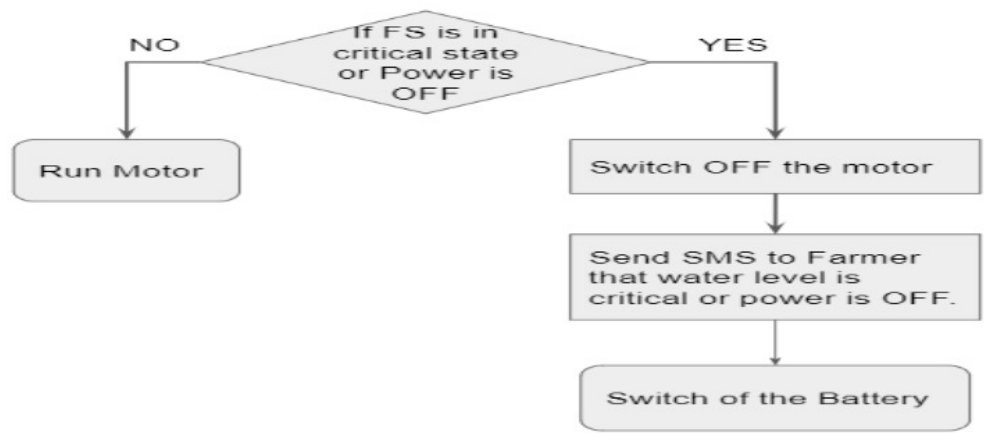

Figure 5: Software flow: (a) flowchart to switch on motor and (b) flowchart to switch off motor. 


\section{Conclusion}

In this paper, we present a AMSU used to get the water level indication in well and remotely control the motor in the farm field using mobile. GSM link is used between the AMSU and mobile. The system is different than the existing systems in that we consider the problems caused due to power cuts. Once the power has came back, the availability of power in the farm field is sent to the farmer mobile along with the water level indication. Such a system reduces the burden on farmer for going to the farm field in order to check the water level in well for turning motor on/off. Through this AMSU, farmers are able to utilize highly detailed analytics to accelerate productivity and gain more access to opportunities in the future. A discussion of current available wireless communication protocols for agriculture applications has also been given in this paper.

\section{Acknowledgements}

Authors would like to thank local farmers in Warangal and Kodad regions for allowing the prototype testing in their farm fields. Authors appreciate the support provided by Department of Electronics and Communication Engineering of Jawaharlal Nehru Technological University Hyderabad, India.

\section{References}

[1] G. S. Nagaraja, A. B. Soppimath, T. Soumya, and A. Abhinith. Iot based smart agriculture management system. In 2019 4th International Conference on Computational Systems and Information Technology for Sustainable Solution (CSITSS), volume 4, pages 1-5, 2019.

[2] H. V. Abhijith, D. A. Jain, and U. A. Athreya Rao. Intelligent agriculture mechanism using internet of things. In 2017 International Conference on Advances in Computing, Communications and Informatics (ICACCI), pages 2185-2188, 2017.

[3] A. D. Kadage and J. D. Gawade. Wireless control system for agricultural motor. In 2009 Second International Conference on Emerging Trends in Engineering Technology, pages 722-725, 2009.

[4] G. Ulaganathan, Azha Periasamy, and Elavazhagan Murugan. Embedded system based submersible motor control for agricultural irrigation using gsm and to prevent it against over loading, dry running and single phasing automatically. 2014.

[5] Tamoghna Ojha, Sudip Misra, and Narendra Singh Raghuwanshi. Wireless sensor networks for agriculture: The state-of-the-art in practice and future challenges. Computers and Electronics in Agriculture, 118:66 - 84, 2015.

[6] C. Park and T. S. Rappaport. Short-range wireless communications for next-generation networks: Uwb, $60 \mathrm{ghz}$ millimeter-wave wpan, and zigbee. IEEE Wireless Communications, 14(4):70-78, 2007.

[7] K. Zhong, X. Zhou, J. Huo, C. Yu, C. Lu, and A. P. T. Lau. Digital signal processing for short-reach optical communications: A review of current technologies and future trends. Journal of Lightwave Technology, 36(2):377400, 2018.

[8] I. Gresham, A. Jenkins, R. Egri, C. Eswarappa, N. Kinayman, N. Jain, R. Anderson, F. Kolak, R. Wohlert, S. P. Bawell, J. Bennett, and J. . Lanteri. Ultra-wideband radar sensors for short-range vehicular applications. IEEE Transactions on Microwave Theory and Techniques, 52(9):2105-2122, 2004.

[9] Chwan-Lu Tseng, Joe-Air Jiang, Ren-Guey Lee, Fu-Ming Lu, Cheng-Shiou Ouyang, Yih-Shaing Chen, and Chih-Hsiang Chang. Feasibility study on application of gsm-sms technology to field data acquisition. Computers and Electronics in Agriculture, 53(1):45 - 59, 2006.

[10] K. S. V. Grace, S. Kharim, and P. Sivasakthi. Wireless sensor based control system in agriculture field. In 2015 Global Conference on Communication Technologies (GCCT), pages 823-828, 2015.

[11] L. Dan, C. Xin, H. Chongwei, and J. Liangliang. Intelligent agriculture greenhouse environment monitoring system based on iot technology. In 2015 International Conference on Intelligent Transportation, Big Data and Smart City, pages 487-490, 2015.

[12] G. Sahitya, N. Balaji, C. D. Naidu, and S. Abinaya. Designing a wireless sensor network for precision agriculture using zigbee. In 2017 IEEE 7th International Advance Computing Conference (IACC), pages 287-291, 2017.

[13] Gu-Zhah Hong and Ching-Lu Hsieh. Application of integrated control strategy and bluetooth for irrigating romaine lettuce in greenhouse. IFAC-PapersOnLine, 49(16):381 - 386, 2016. 5th IFAC Conference on Sensing, Control and Automation Technologies for Agriculture AGRICONTROL 2016. 
[14] R. Kamath, M. Balachandra, and S. Prabhu. Raspberry pi as visual sensor nodes in precision agriculture: A study. IEEE Access, pages 45110-45122, 2019.

[15] P. Singh and S. Saikia. Arduino-based smart irrigation using water flow sensor, soil moisture sensor, temperature sensor and esp8266 wifi module. In 2016 IEEE Region 10 Humanitarian Technology Conference (R10-HTC), pages 1-4, 2016.

[16] A. Khattab, A. Abdelgawad, and K. Yelmarthi. Design and implementation of a cloud-based iot scheme for precision agriculture. In 2016 28th International Conference on Microelectronics (ICM), pages 201-204, 2016.

[17] D. Davcev, K. Mitreski, S. Trajkovic, V. Nikolovski, and N. Koteli. Iot agriculture system based on lorawan. In 2018 14th IEEE International Workshop on Factory Communication Systems (WFCS), pages 1-4, 2018.

[18] Chandrashekhar S. Patil, R.R.Karhe, and M. A. Aher. Review on generations in mobile cellular technology. 2012.

[19] Jan Magne Tjensvold. Comparison of the ieee 802.11, 802.15.1, 802.15.4 and 802.15.6 wireless standards. 2007.

[20] P. Bhagwat. Bluetooth: technology for short-range wireless apps. IEEE Internet Computing, 5(3):96-103, 2001.

[21] T. Adame, A. Bel, B. Bellalta, J. Barcelo, and M. Oliver. Ieee 802.11ah: the wifi approach for $\mathrm{m} 2 \mathrm{~m}$ communications. IEEE Wireless Communications, 21(6):144-152, 2014.

[22] Alexandru Lavric and Valentin Popa. Performance evaluation of lorawan communication scalability in large-scale wireless sensor networks. Wireless Communications and Mobile Computing, 2018:6730719:1-6730719:9, 2018. 